\title{
Atmospheric-PLD as a New Trend for Nanofabrication in Nanotechnology
}

\author{
Taj Muhammad Khan* \\ School of Physics and CRANN, Ireland \\ *Corresponding author: Taj Muhammad Khan, Dublin 2, Ireland, Email: khanta@tcd.ie
}

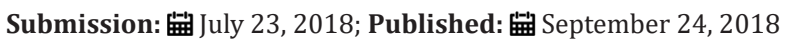

\begin{abstract}
Nanotechnology has emerged as a multidisciplinary technology, feeding all sectors of nanosciences and nanoengineering. Today, smart materials with entirely altered chemical and physical properties and devising new technological methods to produce such materials is on priority of nanotechnology. While working in this direction, recently a new method of atmospheric PLD (APLD) was explored and developed. This method shifts the fabrication of plasmonic nanomaterials into a completely simple, feasible, environmentally friendly and industrial oriented technique. In this newly introduced method, ablation plume produced by a pulse laser of enough fluence at atmospheric gas pressure, is captured by the gas to form a nanoparticle (NP) aerosol by collisional condensation. The NP aerosol is transported by various gas flow or flowing plasma approaches to produce NP film which are directly used as SERS substrates for chemical detection purpose.
\end{abstract}

Keywords: New synthesis methods in material science; APLD; Nanomaterials; NP film; NP aerosol

\section{Introduction}

In this short letter, a new fabrication method is introduced, recently emerged as one of the feasible routes to make plasmonic metal nanomaterial. This method is based on pulsed laser ablation (PLA) of the target material at atmospheric gas pressure. At these conditions, hydrodynamic expansion of the laser produced plasma plume is significantly different from its expansion in vacuum or low-pressure gas. In vacuum or low-pressure gas $(<0.1 \mathrm{mbar})$, ablation plume expands freely away from the target surface compared to atmospheric gas pressure where ambient gas severely restricts plume expansion to $1-2 \mathrm{~m}$. To efficiently utilize the laser ablation plume and the deposition process at atmospheric gas pressure, a novel method of atmospheric PLD (APLD) was recently introduced and applied to produce plasmonic nanoparticle (NP) films $[1,2]$. The NP films produced by this method were effective as SERS substrates used in surface enhanced Raman spectroscopy (SERS) for the chemical detection purpose [1]. Initially, the method was used with ablation in the confined geometry, where the plasma plume was artificially confined in a small region (gap) between the target and the deposited surface to produce a long-lived dense plasma. The method so called ablation in the confined geometry showed strong potential and a single laser shot was sufficient to make a layer of nanoparticles (NPs) [3]. The newly introduced APLD methods in principle is an alternative to classical PLD and marks the upper end of the pressure domain of vacuum PLD examined to date. In APLD, due to ambient gas, ablation plume from the target surface is restricted in the close vicinity above the target surface and forms a NP aerosol by collisional condensation [4]. The produced NP aerosol is entrained in a gas flow or flowing atmospheric plasma to a substrate to build up a NP film. The new APLD method is technologically feasible with strong potential for industrial scale application. To develop this method, it is constantly under investigation in direction of understanding. The method will open a new fabrication route to make plasmonic materials to feed nanotechnology in various sectors which are technologically important.

In conclusion, a new method of APLD was briefly introduced and conversed with the aim to bring it at the research forefront of the research community. Today, the method is under hot debate and successfully applied to make plasmonic nanomaterials. The development of this technique will directly lead to a nanofabrication in an industrial environment with reduced cost. The method strongly demands to devise various approaches to effectively transport a NP aerosol. In a recent research carried-out in School of Physics, Trinity College Dublin (TCD), some multi-fold plasmas and flowing gas techniques were employed to achieve this goal. The method is relatively fast and has strong potential for industrial scale application.

\section{Acknowledgement}

This research work was supported by Science Foundation Ireland under Investigator Project 12/IP/1662. 


\section{References}

1. Khan TM, Mujawar MA, Siewerska KE, Pokle A, Donnelly T, et al. (2017) Atmospheric pulsed laser deposition and thermal annealing of plasmonic silver nanoparticle films, Nanotechnology 28: 445601.

2. Khan TM, Pokle A, Lunney JG (2018) Atmospheric pulsed laser deposition of plasmonic nanoparticle films of silver with flowing gas and flowing atmospheric plasma. Appl Phys A 124: 336.
3. Donnelly T, Lunney JG (2013) Confined laser ablation for single-shot nanoparticle deposition of silver. Appl Surf Sci 282: 133-137.

4. Itina TE, Voloshko A (2013) Nanoparticle formation by laser ablation in air and by spark discharges at atmospheric pressure. Appl Phys B 113(3): 473-478 (c) (i) Creative Commons Attribution 4.0

For possible submissions Click Here

\section{Submit Article}

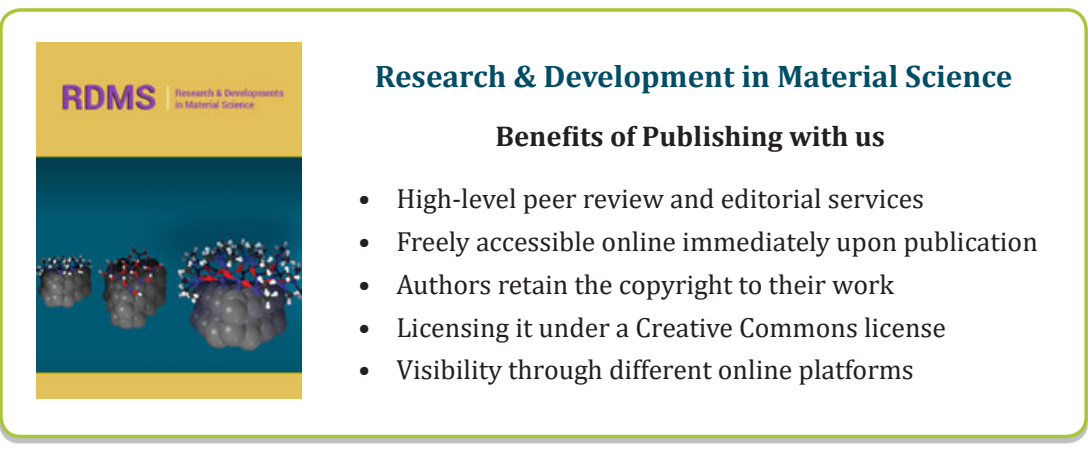

